\title{
Validación de la escala de estereotipos de género en el comportamiento sexual de riesgo en hombres privados de la libertad
}

\author{
Ricardo Sánchez Medina y David Javier Enríquez Negrete \\ Universidad Nacional Autónoma de México (México)
}

\begin{abstract}
El propósito del presente trabajo fue validar la escala de estereotipos de género en el comportamiento sexual de riesgo en población privada de la libertad (PPL). Participaron 687 hombres con una edad promedio de 26 años pertenecientes a un Centro de Reinserción Social de México respondieron una escala de 20 reactivos sobre estereotipos de género sobre la conducta sexual; así como 12 reactivos de la escala de estereotipos de género de Rocha y Díaz-Loving (2005), adicionalmente se comparan las propiedades psicométricas con universitarios de la escala original y se evalúo el uso del condón en relaciones sexuales. Los reactivos se sometieron a un análisis factorial con rotación varimax, se obtuvo el análisis de consistencia interna y la validez convergente, encontrándose propiedades psicométricas adecuadas. Con la escala validada, se encontraron diferencias significativas entre aquellos que usan o no condón en sus relaciones sexuales, aquellos que no lo usan tienen más estereotipos de género. Los resultados se discuten en términos de la importancia de tener instrumentos válidos y confiables en PPL, así como de la influencia de los estereotipos de género en el comportamiento sexual.
\end{abstract}

Palabras clave: validación, estereotipos, uso de condón, entorno carcelario.

Validation of the scale of gender stereotypes in sexual risk behavior in men deprived of their liberty. The purpose of this work was to validate the scale of gender stereotypes in sexual risk behavior in a population deprived of freedom (PDF). A total of 687 men with an average age of 26 belonging to a Social Rehabilitation Center of Mexico participated. They answered a scale of 20 items on gender stereotypes about sexual behavior; as well as 12 reagents of the scale of gender stereotypes of Rocha \& Díaz-Loving (2005), in addition the psychometric properties are compared with university students of the original scale and the use of the condom in sexual relations was evaluated. The reagents were subjected to a factor analysis with varimax rotation, the internal consistency analysis and the convergent validity were obtained, finding suitable psychometric properties. With the validated scale, significant differences were found between those who use or not condom in their sexual relations, those who do not use it have more gender stereotypes. The results are discussed in terms of the importance of having valid and reliable instruments in PDF, as well as the influence of gender stereotypes on sexual behavior.

Keywords: validation, stereotypes, condom use, prison setting.

Correspondencia: Ricardo Sánchez Medina. UNAM, FES Iztacala. Torre Académica, segundo piso, cubículo 20. Av. De los Barrios № 1. Los Reyes Iztacala, Tlalnepantla, Edo. de México (México). E-mail: ricardo.sanchez@ired.unam.mx 
De acuerdo con el Programa Conjunto de las Naciones Unidas sobre el VIH/SIDA (ONUSIDA, 2016) 36.7 millones de personas vivían con el VIH en 2016 en todo el mundo; y 1.8 millones de personas contrajeron la infección por el VIH en el mismo año; siendo las conductas sexuales de riesgo, una de las principales causas de contagio por infecciones de transmisión sexual (ITS). Diversos estudios indican que la práctica del sexo no protegido sigue siendo la causa más importante para adquirir el VIH/SIDA (Paz-Baileya et al., 2016; Sabitu, Iliyasu y Josué, 2009).

Aunado a lo anterior, se indica que el contexto favorece determinados tipos de comportamiento que favorecen el riesgo de infección, uno de estos entornos es el carcelario; en población privada de la libertad (PPL) se ha encontrado que no usan condón en sus relaciones sexuales (Guerrero, 2012), que tienen relaciones sexuales bajo los efectos de sustancias (Chinca, Samalvides, Bernabe-Ortíz, Kruger, y Gotuzzo, 2008) y abuso sexual (Jürgens, Nowak y Day, 2011) y tienen más conductas de riesgo que la población en general.

La literatura muestra que los estereotipos de género tienen un fuerte impacto sobre el comportamiento sexual (Caricote, 2006; García-Vega, Menéndez, García y Rico, 2010). Entendiendo los estereotipos como prejuicios asociados a una visión ideal de la conducta que apuntan a una imagen sesgada del comportamiento de hombres y mujeres (Mercado y Hernández, 2010); los cuales reproducen cierto tipo de relaciones que se relacionan con conductas de riesgo sexual (Caricote, 2006).

Sin embargo, a pesar de que se ha encontrado una relación entre los estereotipos de género sobre la conducta sexual, se ha encontrado que en algunas poblaciones los estereotipos son diferentes (García-Vega et al., 2010). Por ejemplo, en sociedades con una educación machista, existe mayor permisividad cultural, la cual permite a los varones tener múltiples parejas sexuales (Duffy, 2005), de tener relaciones extramaritales (Wood y Price, 1997); generalmente, hombres que consideran que estos comportamientos los hacen mostrar su virilidad, tienden a tener más parejas sexuales (Cianelli, Ferrer y McElmurry, 2008), y que hombres que se encuentran privados de la libertad tienen a presentar más conductas de riesgo (Sánchez, Enríquez y Robles, 2016).

En el caso particular de las mujeres, sus creencias también las pone en riesgo de salud sexual, ya que se les educa para ajustarse a los deseos de su pareja, aceptar la infidelidad y satisfacer sus necesidades sexuales (Cianelli et al., 2008; Wood y Price, 1997); e incluso se ha encontrado que las mujeres consideran que es responsabilidad del hombre decidir el uso del condón en relaciones sexuales (Manji, Peña y Dubrow, 2007).

Como se puede observar los estereotipos de género tienen una relación sobre los comportamientos sexuales de hombres y mujeres; siendo la PPL susceptible a tener conductas de riesgo y a ser más vulnerables socialmente; por lo que es necesario crear programas de intervención orientados a modificar esas creencias estereotipadas que los 
hacen tener comportamiento de riesgo, pero antes de ello es necesario tener los indicadores que permitan evaluar dichas creencias; sin embargo, cuál es el instrumento más idóneo, ya que cada investigación evalúa de manera diferente. Por ejemplo, de acuerdo con la revisión realizada por Calleja (2011) durante un periodo de veinte años sobre cómo se han evaluado los estereotipos en México, encuentra que dichos instrumentos obtienen información referente a los roles, creencias, expectativas y atribuciones sobre cómo son y cómo se relacionan hombres y mujeres, pero de manera genérica. En otras escalas, se comparan hombres y mujeres en términos de las actitudes, creencias o diferencias en el comportamiento sexual, pero que no evalúan las creencias de género en relación con la conducta sexual de riesgo.

Con base en lo anterior, y de acuerdo con la revisión de la literatura, se puede decir, que los estereotipos son diferentes de acuerdo a la población que se esté evaluando, medir los estereotipos en función de las diferencias entre hombres y mujeres en el comportamiento sexual no es suficiente; y finalmente, a pesar de que se ha encontrado relación entre los estereotipos de género y la conducta sexual, generalmente su evaluación está en ámbitos generales y no centrado en creencias estereotipadas sobre la conducta sexual.

Motivo por el cual, el propósito de la presente investigación fue validar la escala de estereotipos de género en el comportamiento sexual de riesgo en hombres privados de la libertad que permita identificar cómo los estereotipos de género en el ámbito sexual influyen sobre la conducta sexual; adicionalmente, se comparan las propiedades psicométricas con universitarios, para mostrar evidencia de cómo los estereotipos de género en el ámbito sexual son diferentes entre diferentes poblaciones.

\section{MÉTODO}

\section{Participantes}

687 hombres privados de la libertad pertenecientes a un Centro de Reinserción Social de la Ciudad de México. A través de un muestreo intencionado (Kerlinger y Lee, 2012) se invitó a participar a todos los internos del centro, accediendo el $45 \%$ de la población total. La edad promedio es de 26.68 años (DE: 3.41 ), el $63.2 \%$ reportó ser solteros, y el porcentaje restante casados o en unión libre. El nivel de estudios de los participantes se distribuyó de la siguiente manera: ninguno (3.3\%), primaria $(21.3 \%)$, secundaria (61.2\%), preparatoria (12.3\%), universidad (1.9\%); más del $84 \%$ se encontraba por el delito de robo.

Adicionalmente se aplicó la escala a 530 universitarios con una edad promedio de 20.69 años $(S D=1.13)$ para comparar los resultados obtenidos en dos muestras. 


\section{Instrumentos}

Para la evaluación de los estereotipos de género en el comportamiento sexual, se utilizó la escala diseñada por Márquez, Enríquez y Sánchez (2017) en población universitaria. Consta de 20 ítems con cinco alternativas de respuesta en una escala tipo Likert (1=totalmente en desacuerdo, y 5=totalmente de acuerdo). Y se aplicó la escala de estereotipos de género en el ámbito interpersonal y social de Rocha y Díaz-Loving (2005), conformada por 12 reactivos en una escala tipo Likert ( $1=$ totalmente en desacuerdo, y $5=$ totalmente de acuerdo), con un alfa de cronbach de .86. Adicionalmente, se preguntó sobre el uso del condón en la última relación sexual (si/no) para comparar los resultados con el instrumento validado.

\section{Procedimiento}

El estudio fue aprobado previamente por las autoridades de cada muestra (dos universidades públicas y del Centro de Reinserción Social). La aplicación fue grupal en aulas proporcionadas por cada institución. Antes de contestar el cuadernillo de evaluación se les informó sobre las consideraciones éticas del estudio haciendo énfasis en la participación voluntaria, el anonimato y que los datos serían utilizados con fines estadísticos.

\section{Análisis de datos}

En las dos muestras se llevó a cabo por separado: el análisis discriminante por reactivo para valorar la sensibilidad del reactivo; posteriormente se llevó a cabo, por ítem, un análisis de la asimetría, de acuerdo a Lloret-Segura et al. (2014) el criterio mínimo aceptable es un valor entre -2 y 2 . Los reactivos que cumplieron con estos criterios fueron considerados para el Análisis Factorial Exploratorio (AFE) con método de componentes principales y rotación Varimax; de los factores extraídos con valor propio mayor de 1, se seleccionaron aquellos reactivos con cargas factoriales mayores de .40 . Con base en los factores se estimó el índice de consistencia interna para la escala. Únicamente en la muestra de PPL se obtuvo la validez convergente con un análisis de correlación $r$ de Pearson; y con el instrumento ya validado se compararon las puntuaciones obtenidas entre aquellos que usaron y no condón en su última relación sexual.

\section{RESULTADOS}

\section{Análisis discriminante}

Se sumaron las puntuaciones de los reactivos que conformaron la escala para obtener el total. A partir de un análisis de frecuencia se obtuvo el cuartil más bajo (percentil 25) y el más alto (percentil 75) para formar dos grupos: 1) Grupo alto y 2) Grupo bajo. Posteriormente se compararon las medias de cada uno de los reactivos de ambos 
grupos por medio de la prueba $t$ de Student para muestras independientes. Si la diferencia resulta no significativa, se elimina el reactivo debido a la falta de sensibilidad del ítem para diferenciar ambos grupos. Los resultados de dicho análisis se presentan en la tabla 1 para la muestra de hombres privados de la libertad y en la tabla 2 para la muestra de universitarios, donde se observa que todos los reactivos fueron significativos, indicando que pueden pasar al siguiente análisis.

Tabla 1. Análisis discriminante por reactivo en la muestra de hombres privados de la libertad

\begin{tabular}{ccccccc}
\hline & \multicolumn{2}{l}{ Grupo bajo $(\mathrm{n}=150)$} & \multicolumn{2}{l}{ Grupo alto $(\mathrm{n}=151)$} & & \\
\hline Reactivo & Media & $D E$ & Media & $D E$ & $t$ & .001 \\
\hline 1 & 1.40 & .742 & 3.28 & 1.43 & 14.265 & .001 \\
\hline 2 & 1.77 & 1.223 & 3.52 & 1.269 & 12.225 & .001 \\
\hline 3 & 1.30 & .599 & 3.20 & 1.296 & 16.293 & .001 \\
\hline 4 & 1.43 & 1.045 & 3.72 & 1.246 & 17.263 & .001 \\
\hline 5 & 1.17 & .599 & 3.40 & 1.307 & 19.008 & .001 \\
\hline 6 & 1.16 & .506 & 3.24 & 1.394 & 17.173 & .001 \\
\hline 7 & 1.38 & 1.028 & 3.72 & 1.190 & 18.270 & .001 \\
\hline 8 & 1.16 & .592 & 3.16 & 1.332 & 16.805 & .001 \\
\hline 9 & 1.29 & .765 & 3.38 & 1.404 & 15.983 & .001 \\
\hline 10 & 1.33 & .748 & 3.34 & 1.248 & 16.884 & .001 \\
\hline 11 & 1.11 & .339 & 3.50 & 1.238 & 22.819 & .001 \\
\hline 12 & 1.11 & .376 & 3.53 & 1.326 & 21.482 & .001 \\
\hline 13 & 1.28 & .706 & 3.25 & 1.395 & 15.403 & .001 \\
\hline 14 & 1.71 & 1.132 & 3.57 & 1.163 & 14.075 & .001 \\
\hline 15 & 1.39 & .903 & 3.65 & 1.292 & 17.595 & .001 \\
\hline 16 & 1.86 & 1.356 & 4.07 & 1.161 & 15.210 & .001 \\
\hline 17 & 1.52 & 1.066 & 3.62 & 1.331 & 15.071 & .001 \\
\hline 18 & 1.11 & .350 & 3.60 & 1.244 & 23.657 & .001 \\
\hline 19 & 1.39 & .802 & 3.65 & 1.190 & 19.273 & .001 \\
\hline 20 & 1.57 & 1.025 & 3.79 & 1.175 & 17.415 & \\
\hline
\end{tabular}

Tabla 2. Análisis discriminante por reactivo en la muestra de universitarios

\begin{tabular}{|c|c|c|c|c|c|c|}
\hline & \multicolumn{2}{|c|}{ Grupo bajo $(n=150)$} & \multicolumn{2}{|c|}{ Grupo alto $(\mathrm{n}=151)$} & \multirow[b]{2}{*}{$t$} & \multirow[b]{2}{*}{$p$} \\
\hline Reactivo & Media & $D E$ & Media & $D E$ & & \\
\hline 1 & 1.21 & .528 & 2.50 & 1.15 & 16.384 & .001 \\
\hline 2 & 1.15 & .409 & 2.33 & 1.09 & 16.221 & .001 \\
\hline 3 & 1.04 & .216 & 2.08 & 1.01 & 16.154 & .001 \\
\hline 4 & 1.04 & .256 & 2.17 & 1.19 & 14.871 & .001 \\
\hline 5 & 1.00 & .061 & 1.74 & 1.12 & 10.496 & .001 \\
\hline 6 & 1.01 & .105 & 1.88 & 1.12 & 12.421 & .001 \\
\hline 7 & 1.00 & .000 & 1.70 & 1.02 & 11.088 & .001 \\
\hline 8 & 1.00 & .000 & 1.53 & .878 & 9.638 & .001 \\
\hline 9 & 1.03 & .181 & 2.21 & 1.18 & 15.997 & .001 \\
\hline 10 & 1.02 & .136 & 2.07 & 1.00 & 16.674 & .001 \\
\hline 11 & 1.04 & .241 & 1.94 & .973 & 14.520 & .001 \\
\hline 12 & 1.01 & .122 & 1.70 & .981 & 11.401 & .001 \\
\hline 13 & 1.02 & .135 & 2.20 & 1.12 & 16.770 & .001 \\
\hline 14 & 1.06 & .289 & 2.62 & 1.05 & 22.992 & .001 \\
\hline 15 & 1.01 & .149 & 2.07 & 1.03 & 16.320 & .001 \\
\hline 16 & 1.09 & .333 & 2.47 & 1.16 & 18.498 & .001 \\
\hline 17 & 1.10 & .415 & 2.66 & 1.17 & 20.140 & .001 \\
\hline 18 & 1.00 & .061 & 1.72 & .879 & 13.012 & .001 \\
\hline 19 & 1.07 & .349 & 2.17 & 1.07 & 15.743 & .001 \\
\hline 20 & 1.28 & .714 & 2.95 & 1.12 & 20.302 & .001 \\
\hline
\end{tabular}




\section{Análisis de la asimetría}

Para evaluar la calidad de los reactivos, se utilizó la valoración de la asimetría, considerando como rango aceptable valores entre -2 y 2 . Los ítems fuera de este rango en la asimetría se eliminan. Se observa en la tabla 3 que para el caso de la muestra de hombres privados de la libertad todos los reactivos están dentro de rango aceptable, en cambio para la muestra de universitarios, únicamente 6 reactivos lo cumplen.

Tabla 3. Valores de la asimetría por reactivo en dos muestras: PPL y universitarios

\begin{tabular}{ccc}
\hline Reactivo & \multicolumn{2}{c}{ Asimetría } \\
\hline & PPL & Universitarios \\
\hline 1 & .625 & 1.244 \\
\hline 2 & .315 & 1.528 \\
\hline 3 & .966 & 2.340 \\
\hline 4 & .497 & 2.110 \\
\hline 5 & 1.061 & 3.887 \\
\hline 6 & 1.161 & 3.245 \\
\hline 7 & .561 & 4.081 \\
\hline 8 & 1.166 & 4.778 \\
\hline 9 & .867 & 1.982 \\
\hline 10 & .696 & 2.254 \\
\hline 11 & .774 & 2.439 \\
\hline 12 & .922 & 4.115 \\
\hline 13 & .851 & 2.190 \\
\hline 14 & .194 & 1.184 \\
\hline 15 & .387 & 2.275 \\
\hline 16 & -.115 & 1.308 \\
\hline 17 & .479 & 1.322 \\
\hline 18 & .734 & 3.474 \\
\hline 19 & .442 & 1.775 \\
\hline 20 & .152 & 0.557 \\
\hline
\end{tabular}

\section{Análisis factorial exploratorio}

Con base en los resultados de la tabla 3, para la muestra de universitarios únicamente para los seis reactivos se aplicó la prueba de esfericidad de Bartlett $(1381.127929, p<001)$ y la medida de adecuación muestral de Kaiser-Meyer-Olkin $(K M O=.813, p<001)$; para el caso de la PPL se sometieron todos los reactivos a la prueba de esfericidad de Bartlett $(4765.837, p<001)$ y la medida de adecuación muestral de Kaiser-Meyer-Olkin $(K M O=.938, p<001)$. Con base en estos resultados, se procedió a realizar el análisis factorial exploratorio.

En la tabla 4 se observan los factores obtenidos en ambas muestras, para el caso de universitarios se obtiene una escala unidimensional que explica el $32.48 \%$ de la varianza con un índice de confiabilidad de .75, mientras que, para la PPL, se obtienen tres factores que explican el $50.75 \%$ de la varianza, con una consistencia interna de .91 . 
SÁNCHEZ y ENRÍQUEZ. Validación de la escala de estereotipos de género en PPL

Tabla 4. Validez de constructo, consistencia interna y porcentaje de varianza explicada

\begin{tabular}{|c|c|c|c|c|c|}
\hline \multirow[t]{2}{*}{ Ítem } & \multirow[t]{2}{*}{ Contenido del reactivo } & \multicolumn{3}{|c|}{$\begin{array}{l}\text { Carga factorial } \\
\text { PPL }\end{array}$} & \multirow{2}{*}{$\begin{array}{c}\begin{array}{c}\text { Carga factorial } \\
\text { universitarios }\end{array} \\
1\end{array}$} \\
\hline & & 1 & 2 & 3 & \\
\hline \multicolumn{6}{|c|}{$\begin{array}{c}\text { Factor 1. Estereotipos de género sobre la responsabilidad y prevención de } \\
\text { riesgos sexuales }\end{array}$} \\
\hline 6 & Solo los varones deben estar informados sobre cómo poner un condón & .687 & & & \\
\hline 5 & La responsabilidad de prevenir un embarazo es exclusiva de la mujer & .668 & & & \\
\hline 13 & Es mal visto que las mujeres planeen un encuentro sexual con su pareja & .660 & & & \\
\hline 8 & En el acto sexual la mujer tiene prohibido solicitar un condón a su pareja & .640 & & & \\
\hline 9 & Es mal visto que las mujeres compren preservativos en la farmacia & .639 & & & 0.506 \\
\hline 11 & La elección de un método anticonceptivo es exclusiva de las mujeres & .630 & & & \\
\hline 12 & $\begin{array}{l}\text { La responsabilidad de cuidar a la pareja del VIH/SIDA es exclusiva del } \\
\text { hombre }\end{array}$ & .609 & & & \\
\hline 18 & $\begin{array}{l}\text { Prevenir riesgos en la salud sexual es responsabilidad exclusiva de los } \\
\text { hombres }\end{array}$ & .600 & & & \\
\hline 10 & Solo las mujeres son capaces de practicar la monogamia sexual & .576 & & & \\
\hline \multirow[t]{2}{*}{7} & $\begin{array}{l}\text { La responsabilidad de proteger a la pareja de una ITS es exclusiva del } \\
\text { varón }\end{array}$ & .480 & & & \\
\hline & Factor 2. Estereotipos de género sobre la confianza en la pareja & & & & \\
\hline 17 & Es mal visto que una mujer le comunique a su pareja su historia sexual & & .459 & & 0.677 \\
\hline 16 & $\begin{array}{l}\text { Cuando una mujer conoce lo suficiente a su pareja puede dejar de usar } \\
\text { condón }\end{array}$ & & .758 & & 0.439 \\
\hline 20 & $\begin{array}{l}\text { Los varones prefieren que sus parejas tomen pastillas anticonceptivas } \\
\text { antes que ellos usar condón }\end{array}$ & & .651 & & 0.676 \\
\hline 15 & $\begin{array}{l}\text { Las mujeres que practican la fidelidad sexual no deberían de usar } \\
\text { preservativo en sus relaciones sexuales }\end{array}$ & & .645 & & \\
\hline 14 & $\begin{array}{l}\text { Las mujeres plenamente enamoradas de su pareja prefieren el coito } \\
\text { interrumpido antes que el condón }\end{array}$ & & .610 & & 0.759 \\
\hline 19 & La educación sexual incita a los hombres a tener relaciones sexuales & & .477 & & 0.631 \\
\hline \multicolumn{6}{|c|}{ Factor 3. Estereotipos sobre masculinidad y comportamiento sexual } \\
\hline 1 & $\begin{array}{l}\text { Solo los hombres cargan condones por si se presenta la oportunidad de } \\
\text { tener relaciones sexuales }\end{array}$ & & & .729 & \\
\hline 2 & $\begin{array}{l}\text { Solo un hombre aceptaría tener relaciones sexuales, aunque falte un } \\
\text { condón para protegerse }\end{array}$ & & & 683 & \\
\hline 4 & $\begin{array}{l}\text { Es más importante en los varones que en las mujeres asear los genitales } \\
\text { antes de tener relaciones sexuales }\end{array}$ & & & .611 & \\
\hline \multirow[t]{5}{*}{3} & Abstenerse de tener relaciones sexuales es solo para mujeres & & & .555 & \\
\hline & $\%$ varianza explicada & 38.26 & 6.56 & 5.92 & 32.48 \\
\hline & Índice de consistencia interna $(\alpha)$ & .889 & .776 & .713 & .754 \\
\hline & Varianza explicada total (PPL): $50.75 \%$ & & & & \\
\hline & Consistencia interna total (PPL): 0.917 & & & & \\
\hline
\end{tabular}

\section{Validez convergente}

A partir de la validación del instrumento con la PPL se relacionaron los puntajes obtenidos de una escala de estereotipos de género en el ámbito interpersonal y social; con la escala validada en el presente estudio sobre los estereotipos de género en el comportamiento sexual, se encontró que los participantes tuvieron puntuaciones altas en ambo instrumentos, indicando que tienen creencias estereotipadas; al hacer un análisis de correlación entre los resultados obtenidos se encontraron diferencias significativas $(r=.693, p<.01$. $)$ 


\section{Diferencias en uso del condón}

Adicionalmente se preguntó sobre el uso del condón en la última relación sexual, formándose dos grupos, posteriormente se diferenciaron entre aquellos que lo usan de los que no, encontrándose diferencias significativas entre los grupos $(t=1.75, p<.05)$, es decir, quienes reportaron más estereotipos de género sobre la conducta sexual son quienes no usan el condón en sus relaciones sexuales.

\section{DISCUSIÓN Y CONCLUSIONES}

El objetivo de la presente investigación fue validar una escala sobre estereotipos de género sobre la conducta sexual; para lo cual se tomó como punto de referencia la investigación de Márquez, Enríquez y Sánchez (2017) quienes evaluaron a través de Redes Semánticas Naturales Modificadas (Reyes-Lagunes, 1993), el significado psicológico de lo que hace un hombre y una mujer en su vida sexual y para prevenir riesgos en una muestra de universitarios; con base en esos resultados se diseñó el instrumento que se validó en la presente investigación.

A groso modo se observa que, para las muestras evaluadas, se obtuvieron propiedades psicométricas adecuadas; por un lado, de acuerdo con Nunnally y Bernstein (1995) existe una buena consistencia interna cuando el valor del Alfa de Cronbach es superior a 0.7, en el caso de los universitarios se encontró un alfa de .754, mientras que para la PPL se obtuvo un índice de .91; por otro lado, se muestra evidencia de que la técnica utilizada de Reyes-Lagunes (1993) es conveniente para el diseño de instrumentos de medición, ya que es sensible a lo que la población de interés piensa y hace.

En términos del análisis factorial exploratorio se encontraron diferencias en las muestras, mientras que para los universitarios se obtuvo un solo factor con seis reactivos, en la PPL se encontraron tres factores con veinte reactivos; esto nos indica que la PPL tiene más creencias estereotipadas en comparación con los universitarios, esto es congruente con otras investigaciones, la muestra evaluada de PPL no todos tienen estudios universitarios y de acuerdo con Rocha y Díaz-Loving (2005) el nivel de estudios influyen en las creencias estereotipadas. El factor que comparten las muestras tiene que ver con la confianza que depositan en la pareja, en términos de cómo el amor y fidelidad influyen para que dejen de protegerse, esto es relevante dado que se presenta evidencia de cómo la confianza en la pareja se asocia con la conducta sexual (Caricote, 2006; Manji, Peña y Dubrow, 2007).

Sin embargo, cuando se trata de las creencias estereotipadas sobre cuestiones de responsabilidad y prevención de riesgos sexuales, la PPL tiene más marcadas estas creencias, ya que consideran que la mujer debe ajustarse a los deseos del hombre, mientras 
que el hombre es quien debe tomar la iniciativa para protegerse sexualmente, coincidiendo con lo que plantea Cianelli et al., (2008) y Duffy, (2005). En cambio, para la muestra de universitarios solo consideran que es mal visto que una mujer compre preservativos en la farmacia. En el factor 3 de la escala validada con PPL, se agrupan creencias estereotipadas sobre la masculinidad y el comportamiento sexual, es decir señalan que los hombres tienden a buscar encuentros sexuales y que la abstinencia es solo para mujeres, manteniendo la idea que hombres tienen que mantener su virilidad y la mujer debe mantenerse virgen (Manji, Peña y Dubrow, 2007; Wood y Price, 1997).

Adicionalmente, se realizó la validez convergente correlacionando las puntuaciones obtenidas entre dos escalas de estereotipos, una en el ámbito social e interpersonal (Rocha y Díaz-Loving, 2005) y sobre el ámbito sexual; en ambas escalas a mayor puntuación indica que tienen creencias estereotipadas; por lo que al comparar las puntuaciones se encontró una relación positiva, es decir, tener creencias de que el hombre es quien mantiene a la familia o que la mujer debe quedarse en casa; también tiene creencias de que el hombre debe proteger a la pareja para evitar infecciones y que la mujer debe abstenerse de tener relaciones sexuales. Por último, se comparó el puntaje obtenido en la escala de estereotipos de género en el terreno sexual y se encontró que aquellos que tienen una visión más estereotipada son quienes no usan condón en sus relaciones sexuales (Manji, Peña y Dubrow, 2007); lo cual muestra la sensibilidad que tiene el instrumento para analizar cómo los estereotipos de género influyen en la conducta sexual, particularmente con el uso del condón. Dentro de las limitaciones del estudio, únicamente se validó la escala con hombres, por lo que es necesario hacerlo con mujeres privadas de la libertad y analizar si se encuentran los mismos resultados o en qué se diferencian. A pesar de que se realizó la validez convergente y de que el instrumento tuvo propiedades psicométricas adecuadas, el siguiente paso es aplicar la misma escala a poblaciones similares y obtener el análisis factorial confirmatorio para tener mayor sustento de su validez y confiabilidad. Sin embargo, pese a ello, la validación de este instrumento puede ser utilizado para tener indicadores de cómo influyen los estereotipos de género sobre la conducta sexual.

\section{Agradecimientos \\ Investigación realizada gracias al Programa UNAM-DGAPA- PAPIITIA304317/IA301616.}

\section{REFERENCIAS}

Calleja, N. (2011). Inventario de Escalas Psicosociales en México 1984-2005. Universidad Nacional Autónoma de México, Facultad de Psicología: Ciudad Universitaria, México.

Caricote, E. (2006). Influencia de los estereotipos de género en la salud sexual en la adolescencia. Educere, 34(10), 463-470. 
Cianelli, R., Ferrer, L., y McElmurry, B.J. (2008). HIV prevention and low-income Chilean women: Machismo, marianismo and HIV misconceptions. Culture, Health \& Sexuality: An International Journal for Research, Intervention and Care, 10(3), 297-306.

Chinca, L., Samalvides, C., Bernabe-Ortíz, A., Kruger, P. y Gotuzzo, E. (2008). Asociación entre el consumo de alcohol y la infección por virus de inmunodeficiencia humana. Revista chilena de infectologia, 25(1), 49-53.

Duffy, L. (2005). Culture and context of HIV prevention in rural Zimbabwe: The influence of gender inequality. Journal Transcultural Nursing, 16(1), 23-31.

García-Vega, E., Menéndez, E., García, P., y Rico, R. (2010). Influencia del sexo y del género en el comportamiento sexual de una población adolescente. Psicothema, 22(4), 606-612.

Guerrero, M. (2012). Conocimientos, actitudes y prácticas sexuales riesgosas ante el VIH/sida en población privada de la libertad. Universidad Nacional de Colombia. Facultad de Medicina. Recuperado de: http://www.bdigital.unal.edu.co/10440/1/597698.2012.pdf

Jürgens, R., Nowak, M. y Day, M. (2011). HIV and incarceration: Prisons and detention. Journal of the International AIDS Society, 14, 26-43.

Kerlinger, F. y Lee, H. (2002). Investigación del comportamiento. Métodos de investigación en ciencias sociales. México: McGraw Hill.

Lloret-Segura, S., Ferreres-Traver, A., Hernández-Baeza, A., y Tomás-Marco, I. (2014). El análisis factorial exploratorio de los ítems: una guía práctica, revisada y actualizada. Anales de Psicología, 30(3), 1151-1169.

Manji, A., Peña, R., y Dubrow, R. (2007). Sex, condoms, gender roles, and HIV transmission knowledge among adolescents in León, Nicaragua: Implications for HIV prevention. AIDS Care, 19(8), 989-995.

Márquez, E.V., Enríquez, D. y Sánchez, R. (2017). Estereotipos de género para el cuidado de la salud sexual en universitarios. Trabajo presentado en el XLIV Congreso Nacional del Consejo Nacional para la Enseñanza e Investigación en Psicología (CNEIP), celebrado del 27 al 29 de abril de 2017 en la Ciudad de Tepic, Nayarit, México.

Mercado, A. y Hernández, A. (2010). El proceso de construcción de la identidad colectiva. Convergencia Revista de Ciencias Sociales, 53, 229-251.

Nunnally, J.C., y Bernstein, I.H. (1995). Teoría psicométrica. México: McGraw-Hill.

ONUSIDA (2016). Hoja informativa. Noviembre de 2016. Recuperado de: http://www.unaids.org/es/resources/fact-sheet

Paz-Baileya, G., Mendoza, M., Finlaysona, T., Wejnerta, C., Lea, B., Rosea, C., Fisher, H. y Prejeana, J. (2016). Trends in condom use among MSM in the United States: the role of antiretroviral therapy and seroadaptive strategies. AIDS, 30(12), 1985-1990.

Reyes-Lagunes, I. (1993). Redes semánticas para la construcción de instrumentos. Revista de Psicología Social y Personalidad, 9(1), 83-99.

Rocha-Sánchez T. y Díaz-Loving, R. (2005). Cultura de género: La brecha ideológica entre hombres y mujeres. Anales de Psicología, 21(1), 42-49.

Sabitu, K., Ilivasu, Z., y Joshua, I.A. (2009). An assessment of knowledge of HIV/AIDS and associated risky behavior among inmates of Kaduna convict prison: the implications for Prevention Programmes in Nigerian Prisons. Nigerian Journal of Medicine, 18(1), 52-58.

Sánchez, R., Enríquez, D., y Robles, S. (2016). Evaluación de un programa de intervención para promover la salud sexual en adolescentes mexicanos en conflicto con la ley. Acta Colombiana de Psicología, 19(2), 257-268.

Wood, M.L., y Price, P. (1997). Machismo and marianismo: Implications for HIV/AIDS risk reduction and education. American Journal of Health Studies, 13(1), 44-52.

Recibido: 20 de mayo de 2017

Recepción Modificaciones: 11 de junio de 2017

Aceptado: 20 de junio de 2017 